Among subtypes, school days missed are depicted in Table 1. Nearly $12 \%$ of children dropped out, 6/25 SoJIA, 1/9 Oligoarticular and 1/14 Enthesitis related arthritis (ERA). Using multivariate linear regression, school absenteeism was found to be significantly affected by physical disability measured by Childhood health assessment questionnaire (CHAQ) $(\mathrm{p}<0.01)$. There was no difference between males and females.

Conclusions Most children missed significant number of school days, highest in SoJIA and least in Polyarticular rheumatoid factor (RF) -ve JIA. Dropout rate was high and related to the JIA subtype. Physical disability significantly affected absenteeism.

\section{PO-1012 EVALUATION OF ASTHMA BIOMARKERS AND PULMONARY FUNCTION TESTS IN CHILDREN WITH ASTHMA}

${ }^{1} \mathrm{~A}$ Tekcan, ${ }^{2} \mathrm{~S}$ Guven, ${ }^{2} \mathrm{D}$ Kuscu, ${ }^{2} \mathrm{~A}$ Yazar, ${ }^{1} \mathrm{E}$ Pala. ${ }^{1}$ Family Medicine, Umraniye Training and Research Hospital, Istanbul, Turkey; ${ }^{2}$ Pediatrics, Umraniye Training and Research Hospital, Istanbul, Turkey

\subsection{6/archdischild-2014-307384.1628}

Asthma is a chronic inflammatory disease of respiratory tract. Many different invasive and noninvasive tests are performed to diagnose asthma. Yet no definite criteria for diagnosing asthma are determined.

Background and aim In our study we aimed to show the relation between blood parameters and pulmonary function tests (PFT) with the asthma severity in patients with a diagnosis of asthma, and post-treatment changes in these parameters.

Method Children aged 0-14 years with asthma seen in our outpatient policlinic were prospectively evaluated. Complete blood count, ECP, specific IgE, Total IgE levels of the patients are recorded at the beginning of the study. Children $>6$ years performed spirometry. All patients filled a study registration form that includes major and minor risk factors. We classified the severity of the disease according to GINA (Global Initiative for Asthma) and we followed the treatment protocol. The severity of the disease did not show any significant correlations with the gender, atopic history and family history.

Results A statistically significant difference was found between the ECP, Total IgE levels and severity of asthma. We observed a decrease in the asthma severity during 2 nd visit.

Conclusion Our results showed that serum ECP levels are significantly correlated with the severity of asthma and may be useful in the assessment of asthma control. Hence the PFT is quite difficult to perform in children, noninvasive parameters are becoming more important for the follow up of the treatment.

\section{P0-1013 CLINICAL AND LABORATORY FEATURES OF CHILDHOOD ATOPIC DERMATITIS}

T Hariyan, O Boyarchuk, N Banadyha. Post-Graduate Institute, Ternopil State Medical Uiversity by Horbachevsky, Ternopil, Ukraine

\subsection{6/archdischild-2014-307384.1629}

Background and aims The aim of the study was to determine the level of ceruloplasmin, oxyproline and cryoglobulinemia of children with atopic dermatitis.

Methods Besides the well-known studies in comprehensive analysis of atopic dermatitis regularities it was defined level of blood ceruloplasmin by Ravin; intensity of metabolism in the connective tissue was evaluated by content oxyproline.
Results Under supervision there were 35 children with atopic dermatitis. In the age aspect younger age group 3 years presented 10 children, and 5 patients were pre-schoolers and children up to 10 years, in puberty to 13 years it was recorded 9 patients and adolescents 14 to 17 years in the observation group got 10 . At the time of admission status of children was assessed as medium to severe in 27 cases and as serious in 8 patients. As a result of children immunoglobulin content with acute exacerbation of AD study it was found that most of the children was observed dysimunohlobulinemia. The examination stated that the average ceruloplasmin, oxyproline several times higher than normal. Higher values were recorded directly they correlate with the activity and severity of atopic dermatitis.

Conclusion It was also revealed typical atopic dermatitis secondary decrease of cellular immunity. Obviously, reducing the activity of cellular immunity is secondary and depends on the period of the disease and its duration, and levels of ceruloplasmin and oxyproline criteria are allergic inflammatory process.

\section{PO-1014 EFFECT OF LASER ACUPUNCTURE ON THE IMMUNOMODULATORY PARAMETERS IN ASTHMATIC CHILDREN AND ITS RELATION TO ASTHMA IMPROVEMENT}

${ }^{1} \mathrm{~N}$ Hassan, ${ }^{2} \mathrm{HH}$ Shaaban, ${ }^{3} \mathrm{IEM}$ Ahmed Kamel. ${ }^{1}$ Researches and Applications of Complementary and Alternative Medicine, National Research Centre, Cairo, Egypt; ${ }^{2}$ Pediatric Medicine, Cairo University, Giza, Egypt; ${ }^{3}$ Researches and Applications of Complementary and Alternative Medicine, National Research Centre, Giza, Egypt

\subsection{6/archdischild-2014-307384.1630}

Background and aims LASER acupuncture has often been recommended as a treatment of asthma. The technique is noninvasive, and suitable for children.

Acupuncture has been shown to have an immediate effect on relieving the symptoms of asthma. Few studies have demonstrated that acupuncture has beneficial clinical and physiologic effects on asthma, including the immunomodulatory effects of inflammatory cells and cytokines. Our aim is to study the effect of low power multichannel laser acupuncture in the improvement of asthmatic children and its relation to changes in their immunomodulatory system (IgE- Interlukin-4).

Methods 20 cases of asthmatic children (7-15years) were subjected to 12 laser acupuncture sessions in acupuncture points selected for asthma according to traditional Chinese medicine 3 times per week. Clinical improvement and medications used were recorded, IL-4, IgE were measured (ELISA technique), Eosinophilic count and pulmonary functions were done before and after sessions.

Results There were a significant improvement in some parameters of pulmonary functions before and after laser acupuncture sessions in comparing to using of conventional medical treatment only (FVC $\mathrm{p}<0.001$, FEV1 $\mathrm{p}<0.001$ ). Also there were improvements in interleukin4 and IgE levels after laser acupuncture sessions (interleukin 4. $\mathrm{p}<0.001, \operatorname{IgE} p=0.002$ ) and there were a significant correlation between some pulmonary functions parameters with IL4 and IgE improvement.

Conclusion Uses of laser acupuncture in accompanied with conventional medical treatment is more effective in controlling of asthmatic children than using conventional medical treatment only and also accompanied with improvement of all IL-4, IgE and pulmonary functions. 